\title{
Use of Amniotic Stem Cells to Promote New Medical Treatments and Advancements in Regenerative Medicine
}

\author{
Brooke Hisrich and Vincent Gallicchio* \\ Professor, Department of Biological Sciences, Clemson University, Clemson- SC 29631, USA
}

\section{Article Info}

\author{
*Corresponding author: \\ Vincent Gallicchio \\ Professor \\ Department of Biological Sciences \\ Clemson University \\ Clemson- SC 29631 \\ USA \\ E-mail: vsgall@clemson.edu
}

Received: April 29, 2017

Accepted: June 6, 2017

Published: June 12, 2017

Citation: Hisrich B, Gallicchio VS. Use of Amniotic Stem Cells to Promote New Medical Treatments and Advancements in Regenerative Medicine. Madridge J Clin Res. 2017; 1(1): 1-9. doi: $10.18689 / \mathrm{mjcr}-1000101$

\begin{abstract}
Copyright: (c) 2017 The Author(s). This work is licensed under a Creative Commons Attribution 4.0 International License, which permits unrestricted use, distribution, and reproduction in any medium, provided the original work is properly cited.
\end{abstract}

Published by Madridge Publishers
Keywords: Amniotic Stem Cells; Regenerative Medicine; Inflammatory Bowel Diseases.

\section{Introduction}

Amniotic stem cells are continuously placed at the forefront of research in the scientific community because of their potential health benefits and advantageous therapeutic uses. Various studies and experiments have been conducted in order to support the hypothesis that amniotic stem cells have pluripotency ability upon induction and have the capability to differentiate into various cell lineages, and importantly, have yet to commit to a specific cell lineage [1]. Because amniotic stem cells have the potential to develop into skin, cardiac, muscle, cartilage, nerve and bone cells/tissue, the possible medical applications are truly plentiful [2]. One major medical application that could alter the entire course of medical treatment is organ regeneration and regenerative medicine as a whole [2]. Amniotic stem cells are safely extracted from the amniotic sac through a medical procedure called amniocentesis. Amniocentesis is a safe and ethical medical procedure that carries little to no harm to the expectant mother or the developing fetus [1].

Amniotic fluid derived stem cells can also be extracted during a C-section very safely without many ethical concerns. Moreover, in various studies, results support the conclusion that human derived amniotic fluid stem cells have the potential to reduce symptoms form inflammatory bowel syndrome and more generally, diminish inflammation from a variety of causes, counteract Dox toxicity that causes cardiac damage, provide crucial clinical information regarding cell responses to pharmacological agents, and much more. The clinical uses seem to be endless with the correct manipulation and treatment of amniotic stem cells and many specific studies and experiments are discussed in detail below in order to support this claim [1].

The major difference between amniotic and embryonic stem cells is embryonic stem cells are pluripotent, meaning they have the potential to differentiate into any type of cell and have not committed to any specific cell lineage. On the contrary, amniotic fluid derived stem cells that come from the amniotic fluid/sac and not from the embryo are multipotent, and while they are capable of differentiating into a few types of cell, they cannot differentiate into any cell lineage like pluripotent stem cells. However, various studies that are discussed in this article have shown amniotic fluid derived stem cells can induce pluripotency. Thus, for the rest of this article, when pluripotency is discussed, it can be assumed the induction of pluripotency is a therapeutically important property [1]. Lastly, an added benefit to using amniotic stem cells for regenerative medicine and therapeutic applications when compared to embryonic stem cells, is that extraction and use of amniotic stem cells is less controversial and is deemed more ethical among medical and scientific professionals [3]. 


\section{Discussion}

\section{Therapeutic Potential of Amniotic Stem Cells for Inflammatory Bowel Diseases}

Two of the most common inflammatory bowel diseases (IBD) are Crohn's disease and Ulcerative Colitis. Both of these IBDs are classified as chronic, reoccurring, inflammatory diseases that impact the gastrointestinal tract. Although complete pathology related to IBDs is unknown, it is known that both environmental and genetic factors can contribute to the diagnosis. Moreover, the current treatment for IBDs is 5-ASA agents and antibiotics preceded by corticosteroids and immunomodulators. If this treatment course is not successful, surgery is considered. Research began to look for alternative treatments for IBDs because of low remission rates for those patients diagnosed with IBDs, in addition to the adaptability to biological agents and low response rates to medication because of side effects on the GI tract.

Mesenchymal stem cells (MSCs) are now considered for an alternative treatment option. Human mesenchymal stem/ stromal cells (hMSCs) are an area of interest because of their appropriate trophic properties that could be used to treat animals diagnosed with IBDs. Researchers were interested in testing if the secreted molecules that derived from second trimester amniotic fluid MSCs (the spindle-shaped MSCs, SSAF-MSCs) could be used as an alternative treatment for inflammatory bowel diseases.

Experimentally colitis was induced by treating mice for 5 consecutive days with dextran sodium sulphate (DSS). Furthermore, the conditioned media (CM) produced and originated from SS-AF-MSCs was delivered intraperitoneally to the DSS treated mice. It was found, after careful observation, that administration of CM, derived from SS-AF-MSCs, helped diminish the gravity of the colitis in the DSS treated mice. Moreover, TGF $\beta 1$ proteins levels significantly increased in the CM treated mice and TNF $\alpha$ and MMP2 protein levels were concurrently reduced. Because the administration of $\mathrm{CM}$ derived from SS-AF-MSCs cells helped reduce the severity of colitis as a whole, it has potential use for treating humans with similar inflammatory bowel diseases. After histopathological examination of the mice's colon region in both the distal and proximal tracts, it was clear $\mathrm{CM}$ treatment that originated from the SS- AF-MSCs cells, significantly reduced the inflammation in comparison to the control group. Moreover, it should be noted that the fresh media did not impact inflammation and remained similar to the DSS-control group [4].

Figure $1 \mathrm{~A}$ represents a section of the colon completely untreated (the negative control with no DSS treatment). Figure $1 \mathrm{~B}$ depicts the extended and inflamed colon five days after the DSS treatment that induced colitis. Figure $1 \mathrm{C}$ is also treated with DSS and $0.5 \%$ fresh media; as stated previously the fresh media did not impact inflammation in any way. Figure 1D was DSS treated with $20 \%$ fresh media. Lastly, Figure $1 \mathrm{E}$ represents the $\mathrm{CM}$-mesenchymal amniotic stem cell derived cells. The reduction in inflammation is quite visible and Figure $1 \mathrm{E}$ most closely resembles Figure $1 \mathrm{~A}$, the control group that does not have colitis. These results and images support CM cells derived from mesenchymal amniotic stem cells have potential therapeutic uses for the treatment of inflammatory bowel diseases such as Crohn's Disease and Colitis [4].

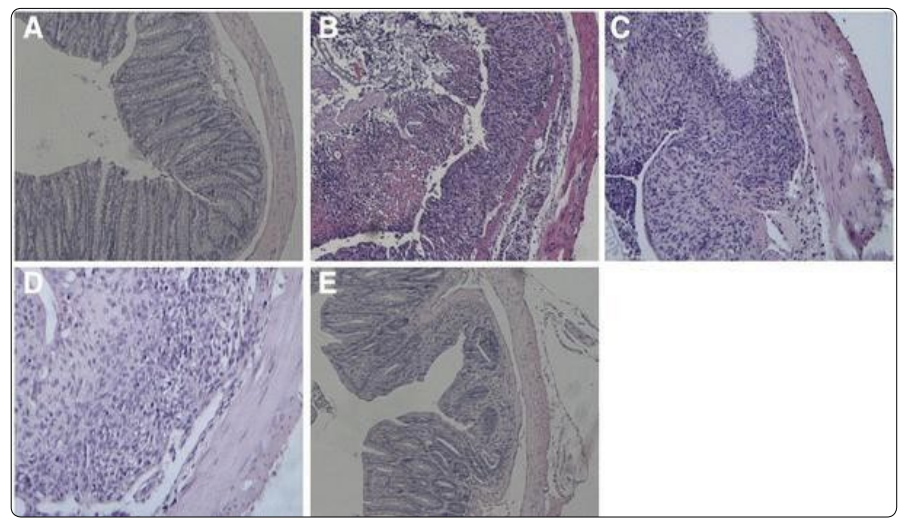

Figure 1. Untreated colon (control), inflamed colon upon induced colitis and colon upon DSS treatment.

Decreased CD90 expression leads augmented differentiation of MSCS

As previously stated, MSCs are mesenchymal stem cells that are considered multipotent, meaning they have the potential to differentiate into many different cell lineages when further developed. Mesenchymal stem cells are characterized as MSCs due to their expression of CD73, CD90 and CD105 cell markers. Specifically in this case, CD90 was primarily considered because its role is still unclear. However, it is known that CD90 is a glycoprotein present in MSC membranes and is also present in some adult and cancer stem cells. In the experiment that is discussed below CD90 was further analyzed in order to gain insight in its function with MSCs [5].

In the experiment, the function of CD90 was analyzed with respect to its proliferation rate, suppression of T-Cell proliferation, morphology and osteogenic/adipogenic differentiation of MSCs. The osteogenic/adiopogenic differentiation of MSCs was investigated through decreasing the expression of a cellular marker that used the CD90-target hairpin RNA vectors. This mutant that decreased the cellular marker that utilized CD90 vectors was significant because it was able to give insight on how differentiation was impacted without fully functioning CD90 [2]. Moreover, osteogenic/ adipogenic differentiation of MSCs is simply the differentiation or development of mesenchymal stem cells into osteocytes and adipocytes [5].

The results of this study depict the clear decline in CD90 expression leads to an augmentation of osteogenic/ adipogenic differentiation from mesenchymal stem cells. Furthermore, these results indicate CD90 may play a role in controlling the differentiation of MSCs, especially the differentiation of osteocytes and adipocytes. CD90 may be functioning in controlling MSCs differentiation by creating an impediment to the committal differentiation pathway that MSCs must overcome in order to differentiate. In vivo the authors believed the differentiation pathway may be 
overcome, even in the presence of CD90, when MSCs are in the presence of the accurate differentiation stimuli. This concept supports the results that imply that altering CD90 levels may allow researchers to gain better and more efficient control on stem cell differentiation in vitro [5].

The potential differentiation was analyzed in two different cell lineage (osteogenic and adipogenic) assays. Non-transduced MSCs, shRNA control MSCs, shRNA CD90 MSCs and CD90negative MSCs were examined in a parallel multilineage assay. Moreover, mesenchymal stem cells were sequestered from dental pulp, amniotic fluid and adipose tissue that was treated with osteogenic differentiation assays [2]. In the osteogenic differentiation assays, mineralized matrix deposition occurred following twenty-one days after the commence of the assay. The mineralized matrix was evaluated first by Alizarin Red Staining with varying calcium concentrations and lastly with examining alkaline phosphatase activity. In Figure 2(a), the staining patterns varied due to differing concentrations of CD90 expression. The shRNA CD90 MSCs presented with much higher staining concentrations because it had higher production of osteogenic differentiation [2], compared to lower staining levels from both non- transduced MSCs and shRNA control MSCs that did not differentiate osteogenically as much as the shRNA CD90 [5].
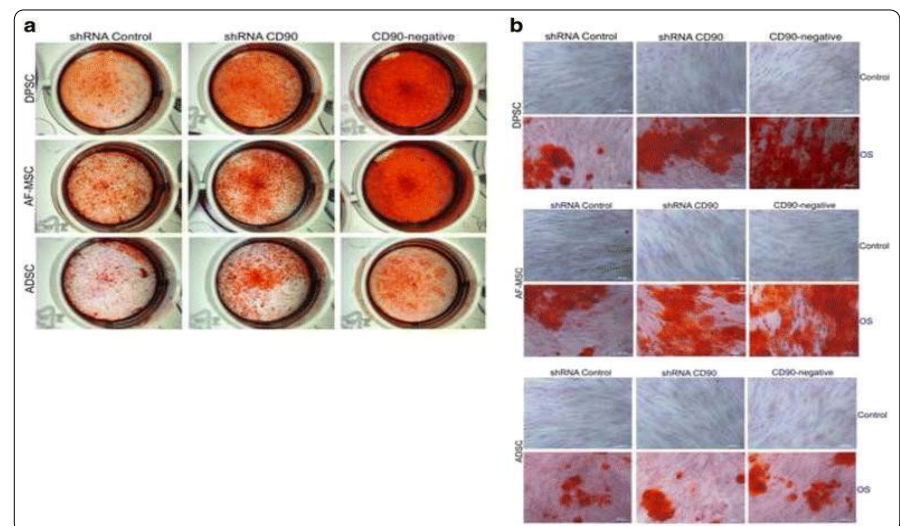

Figure 2: depicts osteogenic differentiation from human amniotic stem cells with different treatment groups, including a control and varying gene markers such as CD90 shRNA MSCs and CD90- neg MSCs.

As previously stated reducing CD90 expression causes an increase in mesenchymal stem cell osteogenic differentiation; leading the authors to conclude that CD90 has regulatory function in the differentiation of MSCs.

Moreover, MSCs, shRNA control MSCs, shRNA CD90 MSCs, and CD90-negative MSCs from amniotic fluid, adipose tissue and dental pulp were all assayed in order to determine their differentiation capabilities in osteogenic cell lineages.

The calcified deposits were then analyzed via the Alizarin Red Staining in order to depict the osteogenic differentiation. The differentiation was observed via gross appearance (Figure 2a) and light microscopy (Figure 2b). Overall, CD90's reduced expression that causes an increase in osteogenic differentiation shines light on CD90's role in stem cell differentiation regulation. This finding can potentially lead to important therapeutic advancements in stem cell medicine [5].

Use of Human Cardiomyocytes for Cardiac Regenerative Medicine in Children
Encountering cardiac progenitor cells could advance clinical stem cell regenerative medicine in treatments of children that are diagnosed with congenital heart disease prenatally. In the following study, researchers examined if functional, transgenefree human cardiomyocytes, also known as CMs, have therapeutic potential application for regeneration after amniocentesis. In order to examine this concept, amniotic fluid specimens were extracted at 20-weeks gestation and the specimens were expanded and reprogrammed to induce pluripotent stem cells. The induction towards pluripotency was done using nonintegrating Sendal virus (Sev) that expressed OCT4, SOX2, CMYC and KLF4. The conditions promoted cardiogenic differentiation after the exposure to induced pluripotency [4]. Furthermore, the amniotic fluid-derived cardiomyocytes (AF-CMs) did generate quite efficiently after being exposed to the specific conditions described above. Following six weeks of exposure, quantitative gene expression depicted that a combined population of differential atrial, ventricular and nodal AF-CMs were generated; this was confirmed after the up-regulation of specific cardiac markers [4]. These specific cardiac markers MYH6, MYL7, TNNT2, TTN and $\mathrm{HCN} 4$ were similar to the quantities expressed by neonatal dermal fibroblast- derived CM controls. AF-CMs had a customary karyotype and revealed the loss of NANOG, OCT4 and the SeV transgene. Including SIRPA+ with AF-CMs revealed a higher efficient and spontaneous beat frequency than the control that consisted of dermal fibroblast; however, the SIRPA+ AF-CMs depicted customary calcium transients and normal chronotropic reactions following the $\beta$-adrenergic agonist Stimulation. Moreover, this data supports somatic cells that exist within the amniotic fluid are capable of being reprogrammed and regenerated to produce functional, transgene-free, $\mathrm{CMs}$ prior to the birth of the child. This data has the potential to lead to the development of new treatments for prenatal cardiac anomalies through the use of regenerative stem cell medicine [6].

Similarities in Response to Pharmaceuticals In Vitro and In Vivo for Amniotic Stem Cells

As previously discussed, it is well known that amniotic stem cells have pluripotential capabilities and manipulating their differentiation could have incredible clinical and therapeutic use. Due to their lack of differentiation and maturation, amniotic fluid stem cells (AFCs) make up a very heterogeneous population of different fetal cells. These fetal cells can be extracted very easily and harmlessly during pregnancy; moreover, this process has been deemed ethical in regards to clinical concerns. In this particular case, specific studies were completed that analyzed the proliferation and differentiation capabilities and multipotency marker and gene expression of amniotic stem cells cultures that were treated with dexamethasone, valproic acid and magnesium sulfate [7].

Upon completion of these studies, similarities and correlations were found between already well-known drug effects on human fetuses and effects on the human amniotic fluid stem cells. Moreover, gene expression analyses supported the clinical findings and results from the study that compared pharmaceutical agents between known effects and effects of the AFCs. The cultures of amniotic fluid stem cells can provide 
important clinical information how they respond to specific pharmacological agents and this new method would provide useful information for how the fetus would respond to those pharmacological agents during pregnancy [7].

\section{Amniotic Stem Cell Secretome counters Doxorubicin-Induced Cardiotoxicity}

Dox, formally known as anthracycline doxorubicin, is used throughout oncology, but it can lead to cardiomyopathy and is not easily treated, cured or prevented. However, the human amniotic fluid stem cells' secretome has been shown to diminish the cardiac damage that can be caused by the use of anthracycline doxorubicin [8].

In the results of this specific study, upon treatment of hypoxic preconditioning, the human amniotic fluid stem cell condition medium can provoke senescence and programmed cell death (apoptosis) of both cardiomyocytes and cardiac progenitor cells. Furthermore, both cardiomyocytes and cardiac progenitor cells are two common characteristics of anthracycline doxorubicin (Dox) cardiotoxicity. A study was completed with mouse ventricular cardiomyocytes and they demonstrated that the inhibition of Dox-senescene and Dox- apoptosis through amniotic fluid stem cell conditioned media lead to overall, significantly decreased DNA damage, the nuclear translocation of NFk $\beta$ and caused an upregulation of specific NFk $\beta$ controlled genes. The NFk $\beta$ controlled genes consist of $/ 16$ and $C x c 11$ and the up-regulation of these genes stimulates mouse neonatal ventricular cardiomyocyte survival [8]. The human amniotic fluid stem cell conditioned media also encourages efflux transporter expression, the efflux transporter is $A b c b 1 b$, and promotes anthracycline doxorubicin extrusion from the mouse neonatal ventricular cardiomyocytes. A signaling cascade, known as PI3K/ Akt, which is upstream of the NFK $\beta$ gene, becomes initiated by the human amniotic fluid stem cell conditioned media and treatment with a P13K inhibitor causes NFk $\beta$ to aggregate into the nucleus, which then prevents Dox from stimulating Dox senescence and apoptosis upon treatment with hAFS-CM [8].

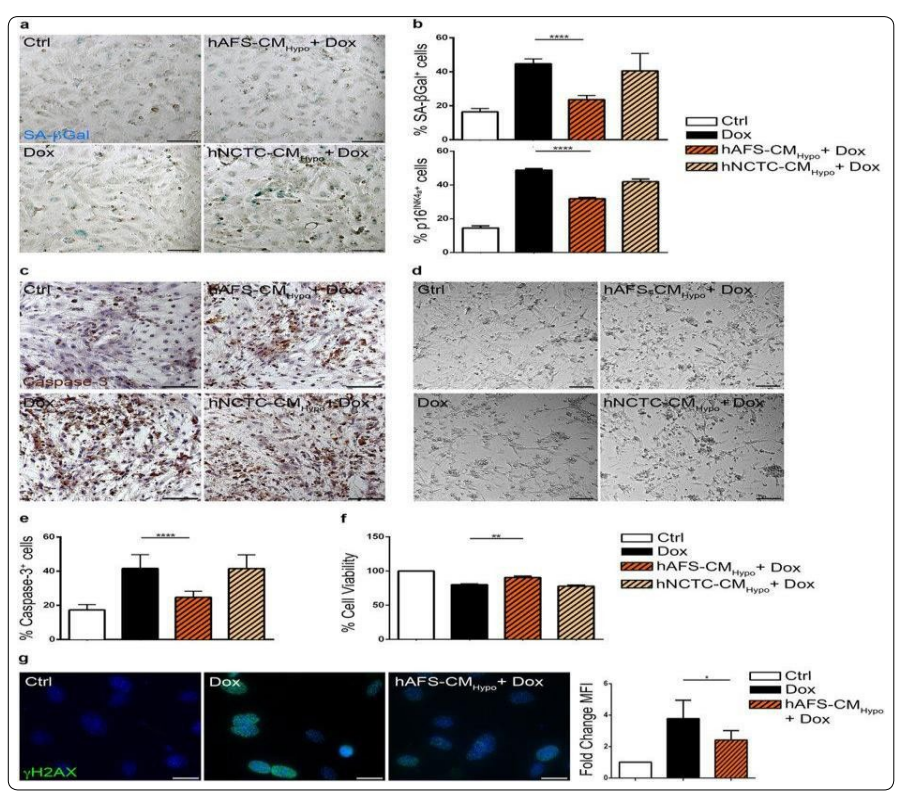

Figure 3A: depicts mNVCMs after stained for senescence with $\boldsymbol{\beta}$-galactosidase (control), varying concentrations of Dox and Dox induced with human amniotic stem cell conditioned media.
Figure $3 \mathrm{~A}$ represents figures of the mouse neonatal ventricular cardiomyocytes after they were stained for senescence-associated $\beta$-galactosidase. Controls were cells untreated with Dox and are represented in the top left corner. The bottom left corner depicts cells stained with $0.2 \mu \mathrm{l}$ of Dox (Dox). Cells were treated with $40 \mu \mathrm{g} / \mathrm{ml}$ of hAFS - CMHypo (hAFS-CMHypo + Dox) or hNCTC-CMHypo (hNCTC-CMNormo + Dox) [8].

Figure $3 \mathrm{~B}$ represents the mouse ventricularcardiomyocytes that expressed senescence-associated $\beta$-galactosidase upon Dox treatment, both with and without the incubation period prior to treatment with $40 \mu \mathrm{g} / \mathrm{ml}$ of the hAFS-CMHypo or the hNCTC-CMHypo. The following results were determined to be: Ctrl: $16.4 \pm 1.9 \%$, Dox: $44.7 \pm 2.9 \%$, hAFS-CMHypo + Dox: $23.6 \pm 2.4 \%$, and hNCTC-CMHypo + Dox: $40.5 \pm 10.2 \%$; ${ }^{* \star *}$ p $<0.0001$. The very small $p$-value is indicative that these experimental results have very little due to chance and that they support the hypothesis that the use of amniotic fluidderived stem cells function to counter the cardiac damage done by Dox. The lower panel of mouse neonatal ventricular cardiomyocytes expressed the p16INK4a following the treatment of Dox, both with and without the incubation period prior to treatment with $40 \mu \mathrm{g} / \mathrm{ml}$ of the hAFS -CMHypo or the hNCTC-CMHypo. The mean and standard deviation is as follows with the following treatments and control: Ctrl: 14.5 $\pm 2.9 \%$, Dox: $48.8 \pm 1.8 \%$, hAFS-CMHypo + Dox: $31.8 \pm 1.5 \%$, and hNCTC- CMHypo + Dox: $41.9 \pm 3.0 \%$. Moreover, the p-value was less than 0.0001 , indicating that the hypothesis is supported and the results have smaller than a $1 \%$ probability of being due to chane [8].

Figure $3 \mathrm{C}$ represents stained mNVCMs for cleaved caspase-3 (Caspase-3), which represent the untreated group that serves as the control. Cells were induced with $1 \mu \mathrm{M}$ Dox $(D o x)$ and cells were induced with hAFS-CMHypo (hAFSCMHypo + Dox) or the hNCTC-CMHypo (hNCTC-CMNormo + Dox) prior to the staining study [8].

Figure $3 \mathrm{D}$ is also representative of the $\mathrm{mNVCM}$ staining and were subjected to aMTT assay, with one control group of untreated cells, cells exposed to $1 \mu \mathrm{M}$ Dox (Dox). The cells were induced with hAFS-CMHypo (hAFS-CMHypo + Dox) or the hNCTCCMHypo (hNCTC-CMNormo + Dox) prior to the staining study [8].

Figure $3 \mathrm{E}$ depicts $\mathrm{mNVCM}$ conveying the cleavedcaspase-3 following Dox treatment both with and without 40 $\mu \mathrm{g} / \mathrm{ml}$ of the hAFS -CMHypo or the hNCTC-CMHypo. The mean and the standard deviation were calculated and expressed on the figure with the results as follows, Ctrl: $17.3 \pm$ 0.8\%, Dox: $41.6 \pm 2.1 \%$, hAFS-CMHypo + Dox: $24.7 \pm 1.1 \%$, and hNCTC-CMHypo + Dox: $41.5 \pm 4.1 \%$. Again the p-value was less than 0.0001 , indicating there was a very small probability that these results were due to chance [8].

Figure $3 \mathrm{~F}$ depicted data from MTT assay, indicating the proportion of sustainable mouse neonatal ventricular cardiomyocytes with the same four treatments, including the one control group. Again, the mean and the standard deviation were calculated and depicted in the figure. The data was as 
follows: Ctrl: 100\%, Dox: $80.0 \pm 0.8 \%$, hAFS- CMHypo + Dox: $90.5 \pm 2.1 \%$, and hNCTC-CMHypo + Dox: $77.8 \pm 1.4 \%$. The $p-$ value was slightly larger, at $p=0.006$ but still small enough for the results to be conclusive that they were not due to chance [8].

Figure 6 depicts images of the mouse neonatal ventricular cardiomyocytes that were stained with $\mathrm{\gamma H} 2 \mathrm{AX}$ for the control group of the untreated cells. The cells that were treated with $1 \mu \mathrm{M}$ Dox (Dox), and cells treated with $40 \mu \mathrm{g} / \mathrm{ml}$ of the hAFS -CMHypo ( $h A F S-C M H y p o+D o x)$. The graph preceding Figure $6 \mathrm{G}$ represents the mean fluorescence intensity (MFI) variation after the Dox treatment, both with and without the hAFSCMHypo. The $p$-value was found to be less than 0.05 and still is small enough to conclude the results were not due to chance. Furthermore, these results support the hypothesis that the secretome from human amniotic fluid derived stem cells helps counteract cardiac damage, such as cardiomyopathy from Dox toxicity. This is yet another clinically therapeutic use of amniotic stem cells that can help better treat patients with various underlying health conditions [8].

\section{Possibility of Neuropathic Pain Alleviation via Amniotic Stem Cells}

The main objective of this study was to analyze the neurobehavior of neuropathic pain caused by chronic constriction injuries also known as $\mathrm{CCl}$ of the sciatic nerve. Chronic constriction injuries cause extreme local inflammation and because of this, the authors thought the use of human derived amniotic fluid stem cells might be able to alleviate some of the neuropathic pain via alleviation of the local inflammation. Specifically in this experiment, the researchers analyzed the potential use of human derived amniotic fluid mesenchymal stem cells; also known was hAFMSCs, for the alleviation of neuropathic pain following a model of chronic constriction nerve injury [9].

The organismal model used for this study was the Sprague-Dawley rat. In order to conduct their study, four 3-0 chromic gut ligatures were used that were loosely ligated about the left sciatic nerve of the organismal model in this case the rat. Following the gut ligatures being ligated and wrapped around the sciatic nerve; intravenous injection of human amniotic fluid mesenchymal stem cells $(5 \times 105$ cells $/ \mathrm{ml})$ was performed. Observations were recorded for three consecutive days [9].

Furthermore, the expression of IL1 $\beta$, TNF $\alpha$ and synaptophysin derived from the dorsalroot ganglion was cultured and then mitigated after it was cultured along with the human derived amniotic fluid mesenchymal stem cells. A reduction of PGP 9.5\% was present in the skin immediately following the chronic constriction injury restitution via the injection of hAFMSCs. Moreover, it was significant that an impactful augmentation of expression of CD68 and TNF $\alpha$ and a reduction in S-100 and neurofilament expression in the nerve that was injured was salvaged all through the human amniotic fluid mesenchymal stem cell injections. Thus, results support that because of the hAFMSC administration the significant increase in synaptophysin and TNF $\alpha$ throughout the dorsal root ganglion could be mitigated and stabilized. Moreover, the neuropathic pain originally felt by an augmentation in the amplitude of sensory action potentials was also attenuated by the administration of hAFMSCs as well. Thus, the human derived amniotic fluid mesenchymal stem cells substantially reduced the neuropathic pain felt due to $\mathrm{CCl}$ and this is supported by the quantitative results from the threshold of mechanical allodynia and the hyperalgesia and the CatWalk XT gait analysis. Moreover, it was concluded by the authors that the human AFMSCs were able to alleviate this pain because they helped reduce the inflammatory response, which in turn altered the neurobehavior that suggested an alleviation of neuropathic pain [10].

\section{Isolation of Amniotic Stem Cells to Differentiate into Osteogenic} and Adipogenic Cells

As previously discussed, human stem cells have shown promise in treating cancer by substituting dysfunctional, cancerous cells with stable, proper functioning cells within a specific target tissue or organ that is the site of the cancer. Stem cells have shown significant potential for regenerative medicine and therapeutic clinical applications; the primary objective of this particular study was to classify and isolate the applicable cell type that would be useful in regenerate medicine studies [11].

The researchers used human amniotic fluid derived stem cells instead have embryonic or bone marrow derived stem cells. Human amniotic stem cells, hAFMSCs, are a great source for pluripotency that have the capability to differentiate into different cell lineages. Isolation of hAFMSCs can be done through extracting both the cell pellet, utilizing the single stage method, or the supernatant of the amniotic fluid. For ethical concerns, researchers have been using excess amniotic fluid in the diagnostic laboratories and it is easier to gain access to, without as many ethical concerns, as extracting embryonic stem cells. In this study, amniotic stem cells were sequestered and cultured on plates for 6 months and utilizing the PCR method and flow cytometry, the cells were replicated and clone at a staggering rate. Moreover, the amniotic stem cells proliferated quite well when cultured and the cells were shown to have differentiated into both osteogenic and adipogenic lineages. Both osteogenic and adipogenic cell lineages were discussed in detail at the beginning of the discussion. Because cultured amniotic fluid stem cells differentiated into osteogenic and adipogenic cells quite successfully, it can be concluded that amniotic fluid is a good source for multipotent amniotic fluid stem cells. Moreover, because it has been shown by multiple researchers that human amniotic fluid derived stem cells are capable of differentiating into multiple cell lineages, this study yet again shows how promising their potential use in the field of regenerative medicine [11].

Once the cultures had differentiated into the osteogenic and adipogenic cells, the authors did a crosstalk between Oct4/NANOG with both ontogenesis and adipogenesis in order to gain better insight into the maturation process. A network analysis was completed using a common targets algorithm and a common regulators algorithim. The network analysis showed data supporting a potential function of MIR302A and MIR let-7-g. The high expression of MIR 302A 
and diminished expression of MIR let-7-g in the human amniotic fluid derived stem cells was shown following the PCR method and flow cytometry after the cells were cultured [11].
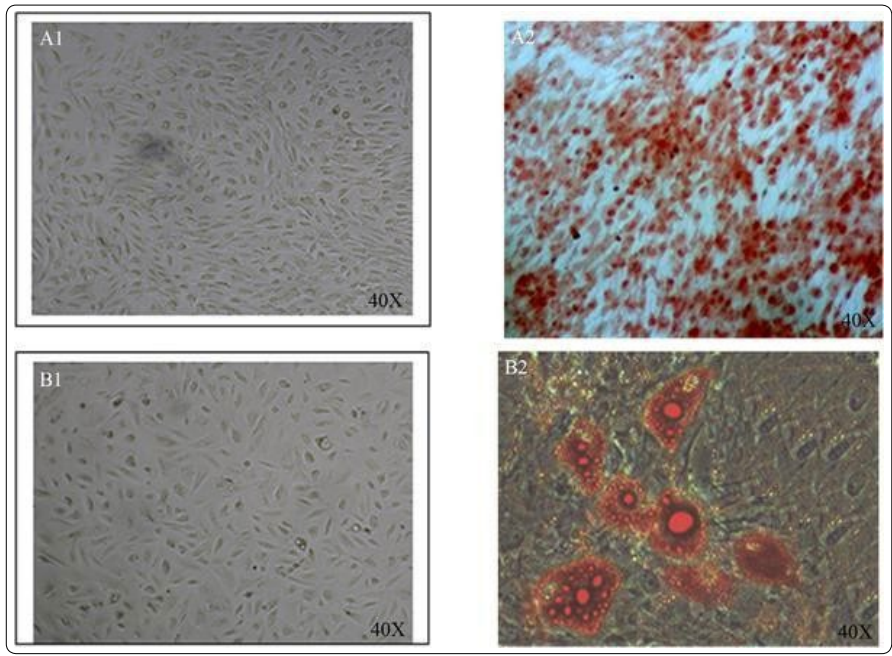

Figure 4: Before and after osteogenic and adipogenic differentiation derived from human amniotic fluid derivedstem cells at $40 x$ magnification.

Figure 4 shows human amniotic fluid derived stem cells that were cultured at $40 \mathrm{x}$ magnification. Figures $4 \mathrm{~A} 1$ and $\mathrm{A} 2$ depict osteogenic cells before and after differentiation. The cells were dyed with alizarin red staining. Figures $4 \mathrm{~B} 1$ and B2 depict adipogenic cells before and after cell differentiation. In Figure 4B2, the adipogenic differentiation shows lipid vacuoles present that clearly differentiate the adipogenic cell type from others. These qualitative images support that human amniotic fluid derived stem cells before and after osteogenic and adipogenic differentiation, supporting that these stem cells have potential uses in regenerative medicine [11].

\section{Amniotic Stem Cell Therapeutic Application: Bone Tissue Engineering}

One main area of particular interest within regenerative medicine is skeletal diseases and the possibility of use of stem cells in order to regenerate bone both in vitro and in vivo. Skeletal diseases are not exclusive and the applications of bone generation could be advantageous to those with degenerative skeletal diseases and/or those caused by trauma, infection or tumors [12].

This study occurred prior to studies as explained beforehand had accounted for hAFMSCs differentiation capabilities, three different stem cell types were examined and their differentiation potentials were analyzed. Adult mesenchymal stem cells, embryonic stem cells and induced pluripotent stem cells all express osteogenic differentiation. Researchers believed amniotic fluid derived stem cells possess advantages for tissue engineering and bone regeneration possibilities due to ethical and practical reasons. As studies earlier in the article have already shown, through the discussion and figures, it has later been proven that these researchers' interest in the use of amniotic stem cells to undergo osteogenesis have been supported through various studies that have successfully induced osteogenesis in amniotic stem cells. Because of this initial research to demonstrate that amniotic stem cells can undergo osteogenesis following differentiation and are supportive, it can be concluded that amniotic fluid derived stem cells are a great model for the field of regenerative medicine. This is also supported because of AFSCs low immunogenicity and lack of tumor maturation after transfection in mice [12].

Inhibition of GSK3 $\beta$ to Differentiate Amniotic Stem Cells into Neural Progenitor Cells

There is evidence that indicates that amniotic fluid derived stem cells are able to differentiate into neural cells given the correct conditions that can induce the differentiation. Nevertheless, until this study, the neural differentiation of human amniotic fluid derived stem cells effectiveness and efficiency has remained low [13].

In this particular study, CD117-postive human amniotic fluid stem cells were isolated and their pluripotent capabilities were analyzed through embryonic body formation. Furthermore, induction of the neural differentiation from the amniotic fluid derived stem cells was completed through the use of neuroectodermal medium. Moreover, this study exposed that the glycogen synthase kinase-3- $\beta$ (GSK3 $\beta$ ) inhibitor was capable of activating proliferation of the CD117postive human amniotic fluid derived stem cells without impacting their uncommitted and immature state. Additionally, SB216763 can encourage neural differentiation of CD117-postive human amniotic fluid derived stem cells to maturate into neural progenitor cells with DMEM/F12 and the N2 supplement present along with SB216763. This finding provides insight on an efficient and cost effective method to induce and maintain proliferation of human amniotic stem cells, along with inducing the differentiation of neural progenitor cells from hAFS cells. Learning more about the induction of neural differentiation from amniotic stem cells may provide possible treatment options for neurodegenerative diseases [13].

\section{Amniotic Mesenchymal Stem Cells have Anti-Cancer Effect on Breast Cancer}

As previously stated, human amniotic stem cells have the capability to self-renew and differentiate into multiple cell lineages due to their multipotent nature. More specifically, they are capable of differentiating into three distinct germ layers. In this study [14] 5-ml of amniotic fluid was obtained from ten 16-20 week pregnant women through amniocentesis. The human amniotic fluid derived stem cells were sequestered and isolated and then the hAFSCs were cultured with T47D breast cancer cells from the specific cancerous cell line. This breast cancer cell line was classified through the use of flow cytometry and PCR, a method also discussed above in a different but similar study. The cultures were observed for five consecutive days and after the third, fourth and fifth day, T47D and the human amniotic fluid stem cells' viability was examined and determined through an MTT assay, this procedure is also discussed above. After five days of being cocultured with human amniotic fluid stem cells, the T47D breast cancerous cells viability remarkably decreased. This result supports the researcher's hypothesis that human amniotic 
fluid derived stem cells are capable of releasing soluble factors into the cell culture with T47D cells, which have an anticancer impact on the breast cancerous cell line [14].

\section{Cases Support Possible Therapeutic Applications for Amniotic Fluid Stem Cells}

In this study, amniotic fluid samples were extracted from 165 pregnant women through amniocentesis and the proliferation rate, karyotype, aging process, pluripotency potential and possibility of differentiation into adipose, bone and cartilage cells from human amniotic fluid derived stem cells were examined. Results depicted that mesenchymal stem cells were successfully sequestered from the amniotic fluid sample and that the amniotic fluid derived stem cells shared similar properties and features of other MSCs. This study was the first that had such large cohort of patients involved and can truly be seen as a reliable and comprehensive study [15].

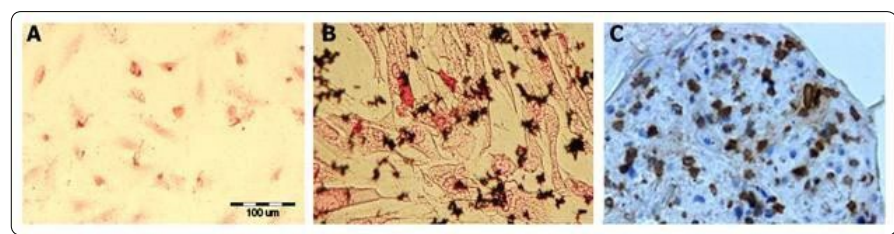

Figure $5(A-C)$ : depicts three boxes of committed, differentiated cells that derived from hAFSCs. Panel A- adipogenic cells, Panel

B- Osteogenic cells and Panel C-Chrondogenic cartilage cells.

Figure $5(A-C)$ depicts successfully differentiated cells from some of the amniotic fluid samples extracted from the 165 pregnant women through amniocentesis. Figure $5 \mathrm{~A}$ represents adipogenesis and shows oil red staining that expresses the lipid cultures present after the amniotic fluid sample was cultured with adipogenic medium for two weeks. Figure $5 \mathrm{~B}$ represents osteogenesis and that von Kossa staining represents the mineralization after the amniotic fluid sample is cultured with osteogenic medium for two weeks. Lastly, Figure $5 \mathrm{C}$ represents the chondrogenic lineage and the immunohistochemistry staining exposes the collagen expression after the amniotic fluid sample was cultured with chondrogenic medium for two weeks [15].

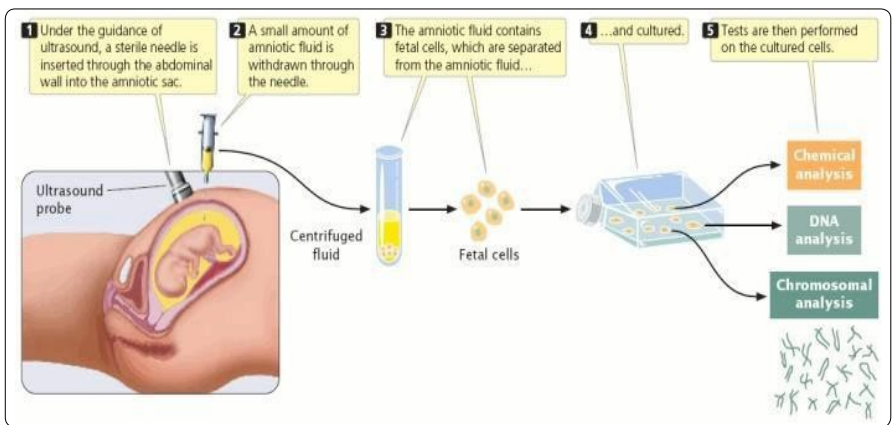

Figure 6: Procedure to obtain, collect and purify amniotic derived mesenchymal stem cells (AMSCs) from pregnant women.

Figure 6 clearly depicts how the amniotic fluid samples are safely extracted from the expectant women and then cultured in order to perform the various tests described above. Moreover, as previously described, one advantage to using amniotic stem cells over embryonic stem cells is the safe and ethical manner in which the samples can be extracted through the amniocentesis [16].
Differentiation of Amniotic Stem Cells into Insulin-Producing Cells

The primary objective of this study is to analyze the capability of human amniotic fluid stem cells to differentiate into insulin-producing cells. In this study, human amniotic fluid derived stem cells were induced to differentiate into pancreatic cells [17]. Through PCR and immunofluorescence, pancreatic genes and proteins were expressed, specifically pancreatic and duodenal homeobox-1 insulin and glucose transporter 2. The insulin that was secreted by the differentiated pancreatic cells was tested through an immunosorbent assay [17]. Moreover, human amniotic fluid derived stem cells were effectively sequestered from the amniotic fluid that contained pluripotent potential stem cells through markers of embryonic stem cells, including mesenchymal stem cells [18]. The amniotic fluid stem cells that contained the pluripotent markers at embryonic antigen 1 were obtained and PCR analysis depicted pancreatic and duodenal homoebox-1 that was present with the box gene 4 and paired box gene 6 , these genes were present early on in the phase of induction and these genes were continued to be expressed in the differ ntiated pancreatic cells [17]. Moreover, the pancreas-related genes, such as insulin, were present in the differentiated pancreatic cells. Immunofluorescence represented that the differentiated pancreatic cells secreted insulin and insulin was able to respond to respond to glucose stimulation. This has potential therapeutic applications for treatment of diabetes mellitus [18].

\section{Mesenchymal Stem Cells Reduce Acuteness of Cisplatin Induced Nephrotoxicity}

Cisplatin is known to be a nephrotoxic that functions as a chemotherapeutic agent. In this particular study, researchers were interested in evaluating if human amniotic fluid stem cells were capable of preventing cisplatin-induced acute kidney injury, also known as AKI. The model organism used was the Sprague-Dawley rat. Eighty rats were used for this study and they were divided into four separate groups with 20 rats in each group.

Group 1 was the saline-injected group and this group was used as the control. Group 2 was the cisplatin-injected group and was used as the positive control. Group 3 was the cisplatin-injected group and the human amniotic stem cells treated group with a cell concentration of $5 \times 106$ for the hAFSCs. The stem cells were administered only a day following the cisplatin-injection. Group 4 contained cisplatin-injections and a culture media treated group. These primary groups were then further divided depending on their time of sacrifice, which occurred 4,7,11 and 30 days following the cisplatin injection. Following sacrifice, renal function tests were completed and the kidney oxidative stress parameters were determined (MDA, SOD and GSH). Moreover, histopathological scoring and regenerative alterations/variations were evaluated [19].

Upon examination, the cisplatin-injected rats had a substantial increase in their serum creatinine and MDA and a reduction in their SOD, GSH and creatinine clearance. The 
alterations occurred as early as four days following the injection and the use of human amniotic stem cells. With only the cisplatin injection, inflammation, tubular necrosis, atrophy and fibrosis were all found. However, with the use of human amniotic stem cells, the injury score significantly decreased after just four days. The researchers concluded that the use of human amniotic stem cells had a protective and regenerative aspect that functioned through antioxidant activity, which ultimately reduced the severity of cisplatin nephrotoxicity [19].

\section{Amniotic Stem Cells Aid in Wound Cell Recovery}

Currently, cellular therapeutic applications often involve the secretion of both growth factors and cytokines. More often than not, growth factors that are secreted are quite limited because they are short lived and do not provide a long-term solution. The primary objective of this study was to improve a hydrogel system that is able to in situ deliver cells that are able to isolate and release growth factors even after the cells that secrete the growth factors are no longer available. The hydrogel that had a fast photocross-linkable heparinconjugated hyaluronic acid (HA-HP) and this was used as the cell delivery vehicle that maintained growth factor release, which ultimately lengthens and sustains paracrine function [20].

In order to analyze the hydrogel in vivo, amniotic fluid derived stem cells, which are known to secrete both cytokines and growth factors, were used on skin wounds in a nu/nu murine model. The results found that the HA-HP hydrogel along with the amniotic stem cells significantly improved wound closure and re-epithelialization as well as augmented vascularization and reproduction of the cellular matrix. Furthermore, this support the hypothesis that the HA-HP hydrogel has the possibility to extend paracrine function of the cells and help augment wound healing in the presence of amniotic stem cells [20].

\section{Summary and Conclusions}

After going into great depth and detail about various therapeutic applications in regards to amniotic stem cell use and research, it is apparent that this area of research can truly alter the course of treatment for many diseases, conditions and the practice of medicine as a whole. The multipotent amniotic stem cells that have induced pluripotent potential are able to differentiate into various cell lineages in order to regenerate particular tissues and restore function that may be impaired due to disease or injury.

Within the discussion of thorough research in regards to amniotic stem cell utilization, it has been shown by multiple researchers in various studies the mesenchymal stem cells, that are derived from amniotic stem cells, have the capability of becoming pluripotent following induction. This induced pluripotent stem cell is able to differentiate into osteogenic, adipogenic, chondrogenic, pancreatic and neural cells, just to name a few of the differentiated cells explored through the various studies discussed.
To summarize specific studies that were discussed, amniotic fluid derived stem cells have shown roles in elevating inflammation in patients suffering from inflammatory bowel disease, showed potential for regenerative function in cardiac conditions through cardiomyocytes, are capable of alleviating neuropathic pain, have anticancer effect in a breast cancer cell line, can differentiate into both pancreatic and neural cells in order to help treat diabetes mellitus and neurodegenerative disorders respectively and much more. Overall, amniotic stem cells are currently one of the most hopeful areas of medicine and research because of their endless possibilities in aiding in therapeutic applications andtreatments for diseases that cover the scope of the entire human body. Through more research and supportive data, regenerative medicine through the use of amniotic stem cells may become a reality for human medical treatments in the future.

\section{References}

1. Bert T. Multipotent Amniotic Stem Cells. Amniotic Stem Cell Injections \& Pluripotency. Hedley Orthopedic Institute. 2014.

2. Cananzi M, Atala A, De P. Stem Cells Derived from Amniotic Fluid: New Potentials in Regenerative Medicine. Reproductive Biomedicine Online. U.S. National Library of Medicine. 2009; 18(1): 17-27.

3. Bani $Y M$, Wilson $P$, Hengstschläger $M$, et al. Amniotic Stem Cells: Potential in Regenerative Medicine. Stem Cells International. 2012. doi:10.1155/2012/530674

4. Legaki E, Roubelakis MG, Theodoropoulos GE, et al. Therapeutic Potential of Secreted Molecules Derived From Human Amniotic Fluid Mesenchymal Stem/Stroma Cells in a Mice Model of Colitis. Stem Cell Rev and Rep. 2016; 12: 604. doi:10.1007/s12015-016-9677-1

5. Moraes DA, Tatiana TS, Lorena FP, et al. A Reduction in CD90 (THY-1) Expression Results in Increased Differentiation of Mesenchymal Stromal Cells. Stem Cell Research \& Therapy. BioMed Central. 2016; 7: 97 doi:10.1186/s13287-016-0359-3

6. Osteogenic Differentiation of Human/Mouse Mesenchymal Stem Cells. R\&D Systems: A Biotech Brand. 2017.

7. Guihua J, Herron TJ, Di Bernardo J, et al. Human Cardiomyocytes Prior to Birth by Integration- Free Reprogramming of Amniotic Fluid Cells. STEM CELLS Translational Medicine. AlphaMed Press, 2016.

8. Dziadosz M, Chan M, Basch R, et al. Effects of Pharmacological Agents on Human Amniotic Fluid-Derived Stem Cells in Culture. Stem Cells and Development. 2016; 25(20): 1510-1579. doi: 10.1089/scd.2016.0141

9. Lazzarini E, Carolina B, Paola A, et al. The Human Amniotic Fluid Stem Cell Secretome Effectively Counteracts Doxorubicin-induced Cardiotoxicity. Nature News. Nature Publishing Group. 2016. doi:10.1038/srep29994

10. Chiang CY, Liu SA, Sheu ML, et al. Feasibility of Human Amniotic Fluid Derived Stem Cells in Alleviation of Neuropathic Pain in Chronic Constrictive Injury Nerve Model. PloS One. U.S. National Library of Medicine. 2016; 11(7). doi:10.1371/journal.pone.0159482

11. Gholizadeh GS, Pashaei AF, Fardyazar Z, Pashaiasl M. Isolation, Characterization, Cryopreservation of Human Amniotic Stem Cells and Differentiation to Osteogenic and Adipogenic Cells. PloS One. U.S. National Library of Medicine. 2016. doi:10.1371/journal.pone.0158281

12. Pantalone A, Antonucci I, Guelfi M, et al. Amniotic Fluid Stem Cells: An Ideal Resource for Therapeutic Application in Bone Tissue Engineering. European Review for Medical and Pharmacological Sciences. U.S. National Library of Medicine. 2016; 20: 2884-2890.

13. Gao $L$, Zhao M, Ye W, et al. Inhibition of Glycogen Synthase Kinase-3 (GSK3) Promotes the Neural Differentiation of Full-term Amniotic Fluidderived Stem Cells towards Neural Progenitor Cells. Tissue \& Cell. U.S. National Library of Medicine. 2016; 48(4): 312-320. doi:10.1016/j. tice.2016.06.001 
14. Ghafarzadeh M, Eatemadi A, Fakhravar Z. Human Amniotic Fluid Derived Mesenchymal Stem Cells Cause an Anti-cancer Effect on Breast Cancer Cell Line in Vitro. Cellular and Molecular Biology (Noisy-le-Grand, France). U.S. National Library of Medicine. 2016; 62(6): 102-6.

15. Bajek A, łgorzata JWO, Walentowicz M, et al. High Quality Independent From a Donor: Human Amniotic Fluid Derived Stem Cells-A Practical Analysis Based on 165 Clinical Cases. Journal of Cellular Biochemistry. Wiley Online Library. 2016; 118(1): 116-126. doi:10.1002/jcb.25618

16. Stem Cells from Amniotic Fluid. InVitro Biotech. 2012.

17. Mu XP, Ren LQ, Yan HW, et al. Enhanced Differentiation of Human Amniotic Fluid-derived Stem Cells into Insulin-producing Cells in Vitro. Journal of Diabetes Investigation.U.S. National Library of Medicine. 2017; 8(1): 34-43. doi:10.1111/jdi.12544
18. Young B, Margaret D. Human Amniotic Fluid: A Source of Stem Cells for Therapeutic Use. American Journal of Obstetrics and Gynecology. Esevier Inc. 2016; 214(3): 321-327. doi:10.1016/j.ajog.2015.12.061

19. Al-Husseiny F, Sobh MA, Ashour RH, et al. Amniotic Fluid-Derived Mesenchymal Stem Cells Cut Short the Acuteness of Cisplatin-Induced Nephrotoxicity in Sprague-Dawley Rats. International Journal of Stem Cells. U.S. National Library of Medicine. 2016; 9(1): 70-78. doi:10.15283/ ijsc.2016.9.1.70

20. Skardal A, Murphy SV, Crowell K, et al. A Tunable Hydrogel System for Long-term Release of Cell-secreted Cytokines and Bioprinted in Situ Wound Cell Delivery. Journal of Biomedical Materials Research. Part B, Applied Biomaterials. U.S. National Library of Medicine. 2016. doi:10.1002/ jbm.b.33736 